\title{
miR-29a Regulates the Proliferation and Migration of Human Arterial Smooth Muscle Cells in Arteriosclerosis Obliterans of the Lower Extremities
}

\author{
Kun Wang Jian Yu Bin Wang Hui Wang Zuolei Shi Guangxin Li \\ Department of Vascular Surgery, Qianfoshan Hospital of Shandong Province, Jinan, China
}

\section{Keywords}

Arteriosclerosis obliterans - microRNA - miR-29a - Human arterial smooth muscle cells .

Platelet-derived growth factor receptor $B$

\begin{abstract}
Background: The molecular mechanisms underlying the contribution of human arterial smooth muscle cells (HASMCs), one of the most important components of the arterial wall, to the pathogenesis of arteriosclerosis obliterans (ASO) remain elusive. Methods: The expression levels of miR-29a in arterial walls were analyzed via real-time-polymerase chain reaction. An ASO cell model was established to investigate the expression of miR-29a on HASMCs. The interaction between miR-29a and platelet-derived growth factor receptor B (PDGFRB) was detected by luciferase reporter assay, and the alteration of the expression of PDGFRB was determined in platelet-derived growth factor-BB (PDGF-BB)-stimulated HASMCs transfected with miR-NC, miR-29a mimics, and miR-29a inhibitors. Further, HASMCs cell proliferation was investigated by cell counting kit- 8 and EdU assays, and cell migrations were evaluated by Transwell and wound closure assays. Results: The expression of miR-29a was remarkably downregulated in the arterial walls of ASO patients compared with normal arterial walls. Furthermore, expression of miR-29a in HASMCs under PDGF-BB stimulation was lower than vehicle control. PDGFRB was identified as a target of miR-29a in HASMCs, and miR-29a inhibited the proliferation and migration in PDGF-BB-induced HASMCs, via regulating the expression of PDGFRB. Conclusion: This study showed that miR-29a is downregulated in the arterial wall of ASO patients, as well as in the PDGF-BB-stimulated HASMCs. This alteration of miR-29a
\end{abstract}

K.W. and J.Y. contributed equally to this work. 


\section{Kidney \\ Blood Pressure \\ Research}

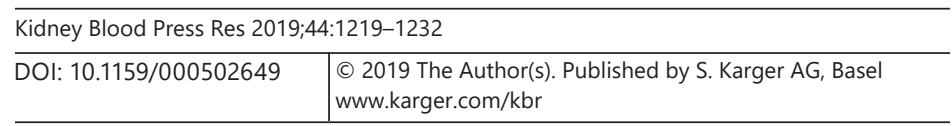

Wang et al.: The Role of miR-29a in ASO

could upregulate target genes PDGFRB and inhibits the proliferation and migration of HASMCs. These findings discovered new mechanisms of ASO pathogenesis, and the miR-29a/PDGFRB axis could serve as potential therapy target of ASO.

\section{Introduction}

Arteriosclerosis is one of the most common vascular diseases particularly among elderly subjects. Human arteriosclerosis obliterans (ASO) is histologically featured by the atherosclerotic thickening and calcification of the arterial walls [1]. Risk factors of ASO include gender, age, hypertension, diabetes mellitus, chronic renal failure, hyperhomocysteinemia, and hyperlipidemia [2]. Notable progress has been made in the treatment of ASO, including surgical techniques, endovascular interventions, and pharmacological treatments. Nevertheless, restenosis following surgery is still limiting the long-term success of artery reconstruction, and ASO remains responsible for the worldwide surgical limb loss [3]. Therefore, early diagnostic methods and new therapies for ASO are required.

MicroRNAs (miRNAs), which are composed of 20-22 nucleotides, are endogenous, noncoding RNAs. miRNAs bind to the 3'UTR of the specific target RNAs via sequence complementarity and inhibit translation and promote mRNA degradation [4]. Many studies have shown that miRNA play significant roles under physiological or pathophysiological conditions including cardiovascular or metabolic disorders [5]. In particular, some studies highlighted the roles of miRNAs in the regulation of ASO. For instance, researchers have identified certain miRNAs as biomarkers in the plasma of ASO patients by microarray analysis and confirmed that miR-4306, miR-4463, and miR-221-3p were significantly downregulated in the plasma and sclerotic samples of ASO patients [6]. Previous study has shown that the expression of miR-142-3p is decreased in CD $4^{+} \mathrm{T}$ cells from ASO patients, which increases the migration of $\mathrm{CD}^{+} \mathrm{T}$ cells to the vascular walls, then regulates actin cytoskeleton [1]. It has been also suggested that miR-31/MFN2 axis plays important role in the pathology of ASO, by promoting the proliferation and migration of human arterial smooth muscle cells (HASMCs) [7].

The human miRNA-29 family includes 3 members, miR-29a, miR-29b, and miR-29c. These 3 molecules differ by 2 or 3 bases [8]. miR-29s has been shown to play critical role in tumorigenesis and cancer progression in multiple cancer types [9]. For example, the expression of miR-29a-3p is related to the effects of Xeroderma pigmentosum D on the proliferation and migration of hepatocellular carcinoma cells, and platelet-derived growth factor B (PDGF-B) was identified as one of the direct targets of miR-29a-3p [10].

Given the important role of miR-29a in the endovascular conditions $[11,12]$, the present study was designed to investigate whether miR-29a is involved and correlated in the pathogenesis of ASO, and HASMCs will be used to establish the in vitro ASO model to investigate the regulation of miR-29a on the proliferation and migration of HASMCs. Further, the direct target of miR-29a in HASMCs will be identified and verified in the cell models.

\section{Materials and Methods}

\section{Clinical Sample Collection}

The clinical protocol to collect samples from ASO patients was approved by the Institutional Review Board of the Qianfoshan Hospital of Shandong Province, and informed consent was obtained from all participants prior to the arteriotomy. Between March 2017 and May 


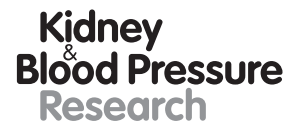

Research \begin{tabular}{l|l}
\hline Kidney Blood Press Res 2019;44:1219-1232 \\
\hline DOI: 10.1159/000502649 & $\begin{array}{l}\text { @ 2019 The Author(s). Published by S. Karger AG, Basel } \\
\text { www.karger.com/kbr }\end{array}$ \\
\hline
\end{tabular}

Wang et al.: The Role of miR-29a in ASO

2018, 26 arterial specimens were collected from ASO patients, and 20 arterial specimens were collected from matched healthy donors. Baseline characteristics of subjects were shown in online supplementary Table S1 (see www.karger.com/doi/10.1159/000502649 for all online suppl. material). All specimens were snap-frozen in liquid nitrogen and stored at $-80^{\circ} \mathrm{C}$ for subsequent use. All samples were identified by 3 independent vascular surgeons in the department.

\section{RNA Extraction and Quantitative Real-Time Polymerase Chain Reaction}

Total RNA was extracted using Trizol reagent (Life Technology, Carlsbad, CA, USA). RNA was reverse transcripted using a miRNA RT kit (TaKaRa Biotech, Dalian, China) according to the manufacturer's instructions. The cDNA was used for quantitative polymerase chain reaction (qPCR) analysis using the SYBR primeScript miRNA real-time PCR kit (Takara) according to the manufacturer's protocol. In addition, qPCR was performed to detect the expression of platelet-derived growth factor receptor $B$ (PDGFRB) using the following primers: PDGFRB-F, 5'-GGCTACATGGACATGAGCAAGG-3', PDGFRB-R, 5'-AGCTTAGCACTGGAGACTCGTTGA-3'. Expression of GAPDH was used as internal control by using primer pair GAPDH-F, 5' ${ }^{\prime}$-ACCGTCAAGGCTGAGAAC-3' and GAPDH-R, 5'-TGGTGAAGACGCCAGTGG-3'. Relative expression level was analyzed using the $2^{-\Delta \Delta \mathrm{Cq}}$ method. All experiments were performed in triplicate.

\section{Western Blotting Assay}

The arterial samples harvested from donors were processed on ice in a lysis buffer (Cell Signaling Technology, Danvers, MA, USA) for $15 \mathrm{~min}$. The lysed samples were centrifuged in $10,000 \mathrm{~g}$ at $4^{\circ} \mathrm{C}$ for $15 \mathrm{~min}$. Twenty-five microgram of protein was loaded and separated by $12 \%$ SDS-PAGE and transferred onto a PVDF membrane (EMD Millipore, Billerica, MA, USA). The membranes were blocked with 5\% nonfat milk in TBS buffer at room temperature for $2 \mathrm{~h}$ and incubated with a mouse anti-PDGFRB monoclonal antibody (1:1,000; Life Technology) at $4{ }^{\circ} \mathrm{C}$ overnight. The membrane was washed 5 times with TBST and incubated with the secondary antibody for $1 \mathrm{~h}$ at room temperature. All membranes were detected using enhanced chemiluminescence (Thermo Fisher Scientific), and protein expression was analyzed using ImageJ (National Institutes of Health, Bethesda, MD, USA).

\section{HASMCs Cell Culture and Platelet-Derived Growth Factor-BB Stimulation}

With informed consent, the primary HASMCs were obtained from the femoral arterial walls of amputation from patients as previously described [13]. ASO cell model was established by stimulating the collected HASMCs by platelet-derived growth factor-BB (PDGF-BB, R\&D Systems, Minneapolis, MN, USA). The cells were cultured in Dulbecco's modified Eagle's medium (Thermo Fisher Scientific) supplemented with 10\% FBS in cell incubator with 5\% $\mathrm{CO}_{2}$ at $37^{\circ} \mathrm{C}$. Relative miR-29a expression in PDGF-BB-treated HASMCs was detected by qPCR.

Transient Transfection with Synthetic miRNAs

The HASMCs cells were transfected with $50 \mathrm{nmol} / \mathrm{L}$ of miR-29a mimics, miR-NC and miR-29a inhibitors, si-PDGFRB (Sangon Biotech, Shanghai, China) in 24-well plates using Lipofectamine in Opti-MEM medium (Thermo Fisher Scientific, CA, USA) according to the manufacturer's manuals. After $24 \mathrm{~h}$, the cells were harvested for RNA and protein extractions to analyze the relative expression of PDGFRB at protein and mRNA levels as described earlier. Following the transfection of miR-29 mimics in HASMCs, PDGF-BB was further used to stimulate the cells, and expressions of miR-29a were compared among all groups. 


\section{Kidney \\ Blood Pressure \\ Research}

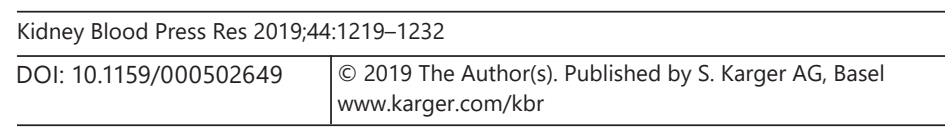

Wang et al.: The Role of miR-29a in ASO

\section{Luciferase Reporter Assay}

The putative target sites of hsa-miR-29a on the 3'-UTR of PDGFRB were predicted using TargetScan online software. HEK293T Cells $\left(5 \times 10^{4}\right.$ cells/well $)$ were cultured in a 24-well plate and cotransfected with wild type (PDGFRB-WT) or mutant (PDGFRB-Mut), miR-29a mimic and miR-NC using Lipofectamine 2000 (Invitrogen). After $48 \mathrm{~h}$, the cells were lysed, and the luciferase activities were measured by the dual-luciferase reporter assay system (Promega, Madison, WI, USA) according to the manufacturer's instructions.

\section{Cell Proliferation Assay}

The regulation of miR-29a on the cell proliferation of PDGF-BB-stimulated HASMCs was measured by EdU and cell counting kit-8 (CCK-8) assays. Briefly, HASMCs were seeded at a density of $5 \times 10^{3}$ cells per well into 96 -well plates. Cells were incubated at $37^{\circ} \mathrm{C}$ for $24 \mathrm{~h}$ following transfection, PDGF-BB at $20 \mathrm{ng} / \mathrm{mL}$ was added to the culture. For the EdU assay, the cells were incubated at $37^{\circ} \mathrm{C}$ in EdU (RiboBio Co., Ltd., Guangzhou, China) solution at 50 $\mathrm{nmol} / \mathrm{L}$ for $2 \mathrm{~h}$ and fixed in $4 \%$ formaldehyde for $30 \mathrm{~min}$ at room temperature. The cells were then incubated with 1,000 $\mu \mathrm{L}$ Cell-Light ${ }^{\mathrm{TM}}$ EdU Apollo ${ }^{\circledR} 488$ (RiboBio) for $30 \mathrm{~min}$, and the nuclear staining with DAPI was $30 \mathrm{~min}$. The fluorescence intensity was captured and analyzed by inverted microscope (magnification, $\times 100$ ). For the CCK- 8 assay, cells in each well were incubated with $10 \mu \mathrm{L}$ CCK-8 solution (Dojindo, Kumamoto, Japan) for $3 \mathrm{~h}$ at $37^{\circ} \mathrm{C}$, and the absorbance was measured at $450 \mathrm{~nm}$.

\section{Cell Migration and Wound Closure Assays}

Cell migration was assessed by Transwell assays. Following transfection with miR-29a and miR-NC, HASMCs cells were suspended in serum-free medium and $100 \mu \mathrm{L}$ cell suspension was added in the upper chamber of each insert (Corning, NY, USA), and the lower chamber was filled with $500 \mu \mathrm{L}$ serum-free Dulbecco's modified Eagle's medium with or without PDGF-BB $(20 \mathrm{ng} / \mathrm{mL})$. After $24 \mathrm{~h}$ of incubation, the migrated cells on the lower surface of the insert were fixed, stained with $0.2 \%$ crystal violet, and counted under a light microscope (Olympus, Tokyo, Japan).

For the wound closure assay, the HASMCs were added into 12 -well plates at $1 \times 10^{4}$ cells per well, and a sterilized pipette tip was used to generate scratch wounds in each well. The scratch wounds were measured after $24 \mathrm{~h}$ using a live cell imaging system (magnification, $\times 100$ ). Image Pro Plus software was used to measure the widths of the scratch and calculate the wound closure percentage.

\section{Statistical Analysis}

The data are presented as the mean \pm SD. One-way analysis of variance followed by a Tukey's post hoc test, or Student $t$ test was used to analyze the data. The data analysis was performed with SPSS 20.0 version (SPSS, Inc., Chicago, IL, USA). $p$ value $<0.05$ was considered to be statistically significant.

\section{Results}

Downregulated miR-29a Expression and Upregulated PDGFRB Expression in ASO

Patients

The expression levels of miR-29a in the arterial walls of ASO and normal donors were analyzed via real-time-PCR. As shown in Figure 1A, miR-29a expression was significantly downregulated in the ASO arteries compared with the normal arteries $(p<0.01)$. Further, we found that the expression level of miR-29a in the media of ASO specimens was significantly 


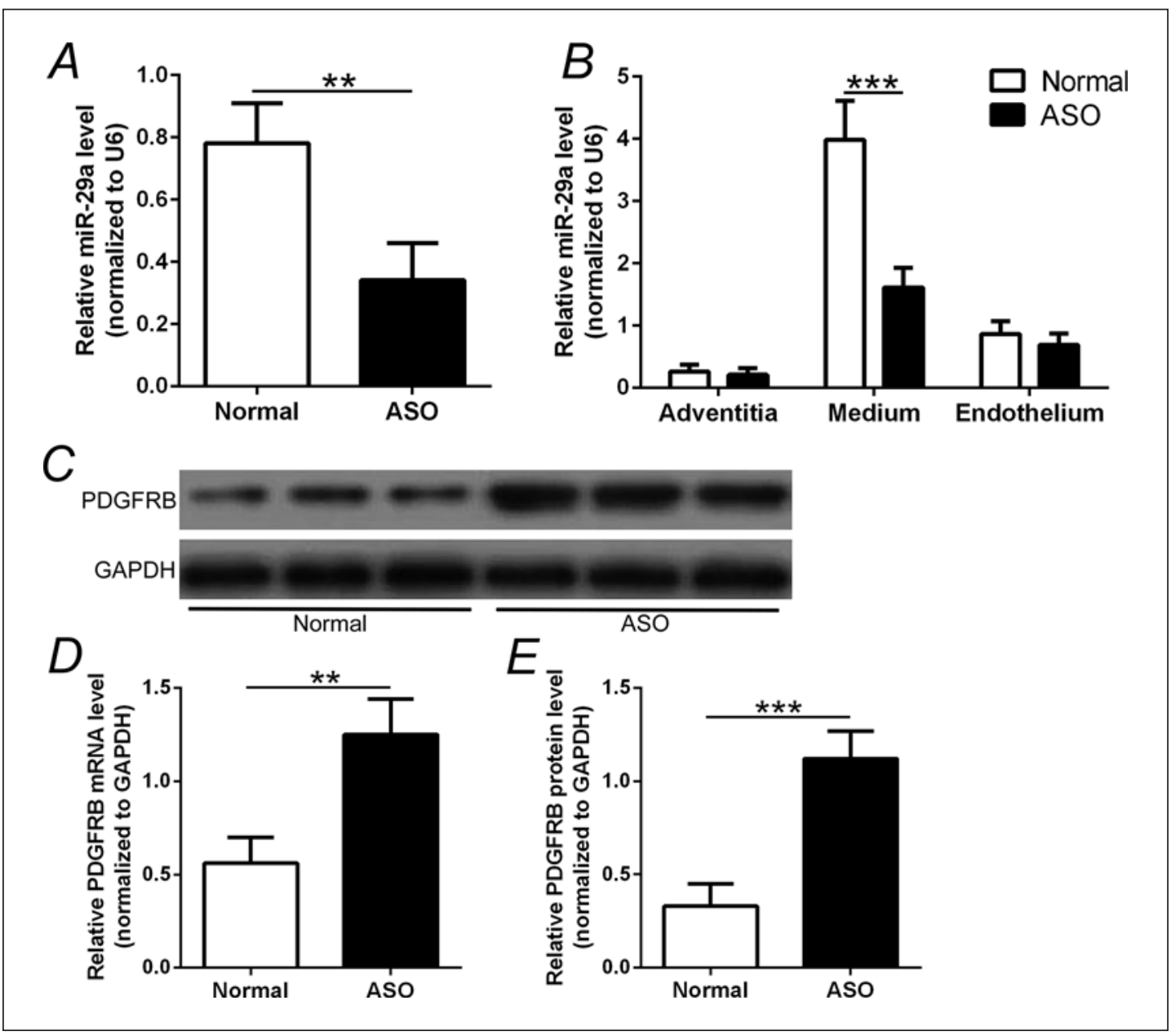

Fig. 1. Downregulated miR-29a expression and upregulated PDGFRB expression in patients with ASO of the lower extremities. A qRT-PCR demonstrates that miR-29a expression is lower in ASO arteries compared with normal arteries. B miR-29a highly expresses in the media of ASO arteries compared to adventitia and endothelium, as assessed by real-time PCR. D qRT-PCR demonstrates that PDGFRB mRNA expression is higher in ASO arteries compared with normal arteries. C, E Western blotting was used to measure the PDGFRB protein expression in ASO arteries compared with normal arteries. Data are presented as mean \pm SD. ${ }^{* *} p<0.01$ and *** $p<0.001$ between the comparison. ASO, arteriosclerosis obliterans; PDGFRB, platelet-derived growth factor receptor $B$.

lower compared with that of the normal control $(p<0.001)$. In contrary, the expressions of miR-29a in the adventitia and endothelium did not show significant differences between normal and ASO arteries (Fig. 1B). Strikingly, real-time-PCR showed that the relative expression of PDGFRB in the ASO arteries was significantly higher than the one of normal arteries ( $p<0.01$, Fig. 1C). This higher expression of PDGFRB in ASO arteries was verified by Western Blot (Fig. 1D, E, $p<0.001$ ). Notably, there was no significant difference of the baseline characteristics of subjects between ASO and normal donors (online suppl. Table S1), which included age, male gender, BMI, smoking history. However, compared to the normal group, the ASO patients had higher percentage of hypertension ( 57.7 vs. 10\%), or diabetes (46.2 vs. $20 \%$ ). These results indicated that miR-29a expression was remarkably downregulated in human ASO arterial walls when compared to the normal arterial walls. 


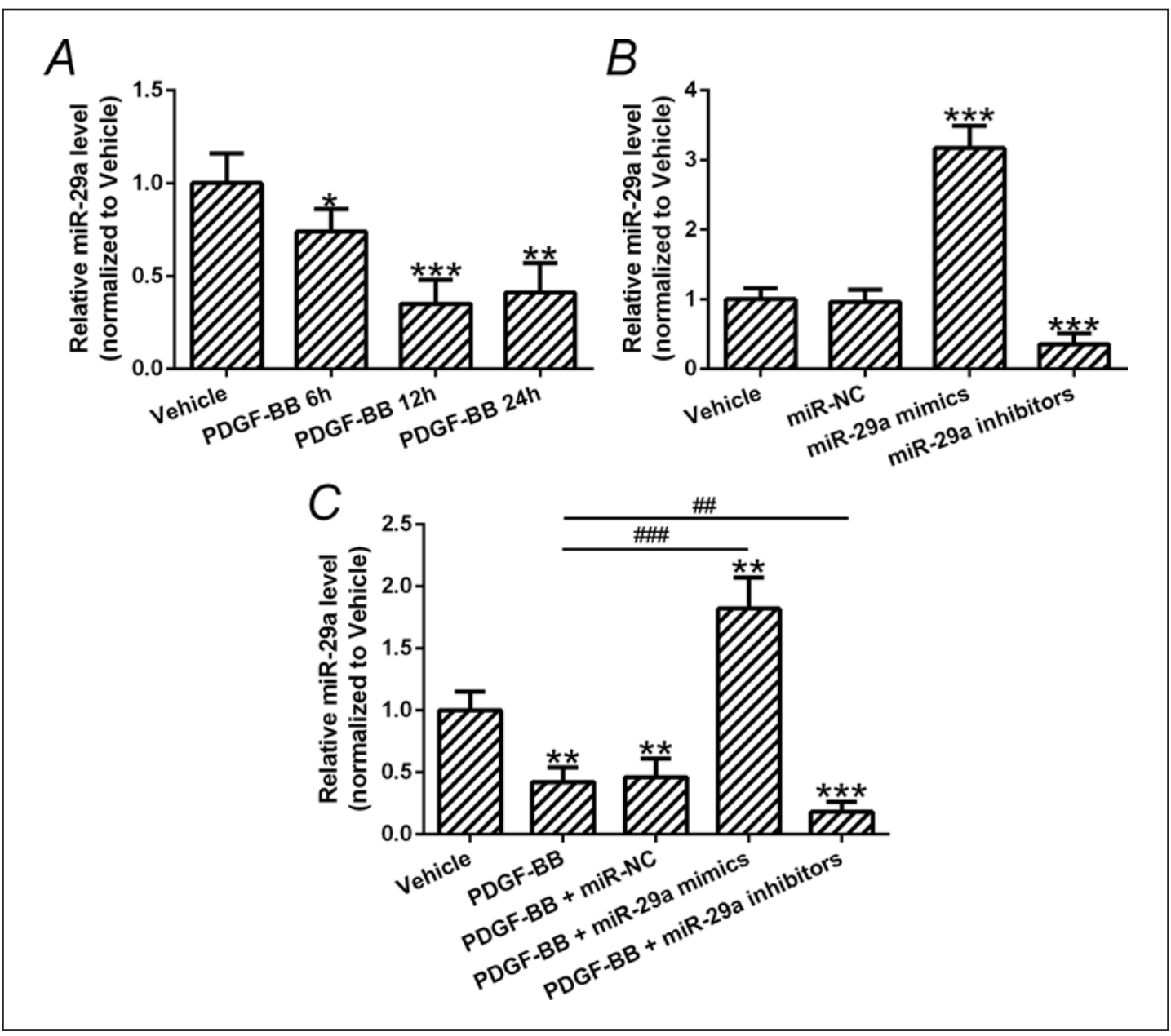

Fig. 2. Expression of miR-29a in HASMCs under PDGF-BB stimulation. A The expression of miR-29a in HASMCs under PDGF-BB stimulation was detected by qRT-PCR. B The expression of miR-29a in HASMCs subjected to miR-29a mimics or miR-29a inhibitors transfection. C The expression of miR-29a in HASMCs transfected with miR-29a mimics or miR-29a inhibitors for $24 \mathrm{~h}$, followed by PDGF-BB stimulation for $24 \mathrm{~h}$. Data are presented as mean $\pm \mathrm{SD}$. ${ }^{*} p<0.05,{ }^{* *} p<0.01$ and ${ }^{* * *} p<0.001$ compared to control. ${ }^{\# \#} p<0.01$, $\# \#$ \#\# 0.001 between the comparison. PDGF-BB, platelet-derived growth factor-BB.

Expression of miR-29a in HASMCs under PDGF-BB Stimulation

Furthermore, an in vitro ASO cell model was established to investigate the expression of miR-29a on HASMCs. As demonstrated in Figure 2A, compared to the vehicle, PDGF-BB-stimulated HASMCs had significantly lower expression of miR-29a. Specifically, the relative expression of miR-29a was significantly lower at $6 \mathrm{~h}(p<0.05)$ and reached the lowest level at $12 \mathrm{~h}(p<0.001)$, and this low level remained until $24 \mathrm{~h}(p<0.01)$ post PDGF-BB stimulation. To further validate the sensitivity of our methods in the determination of miR-29a expression, HASMCs were transfected with miR-29a control oligos (miR-NC), mimics, and inhibitor. We observed that the transfection of miR-NC did not change the expression of miR-29a in the HASMCs, while miR-29a mimics transfected cells showed significantly higher expression of miR-29a (Fig. 2B, $p<0.001$ ), and miR-29a inhibitors transfected cells showed significantly lower expression of miR-29a $(p<0.001)$. Interestingly, we found the transfection of miR-29a mimics could inverse the downregulation of miR-29a expression in HASMCs treated by 


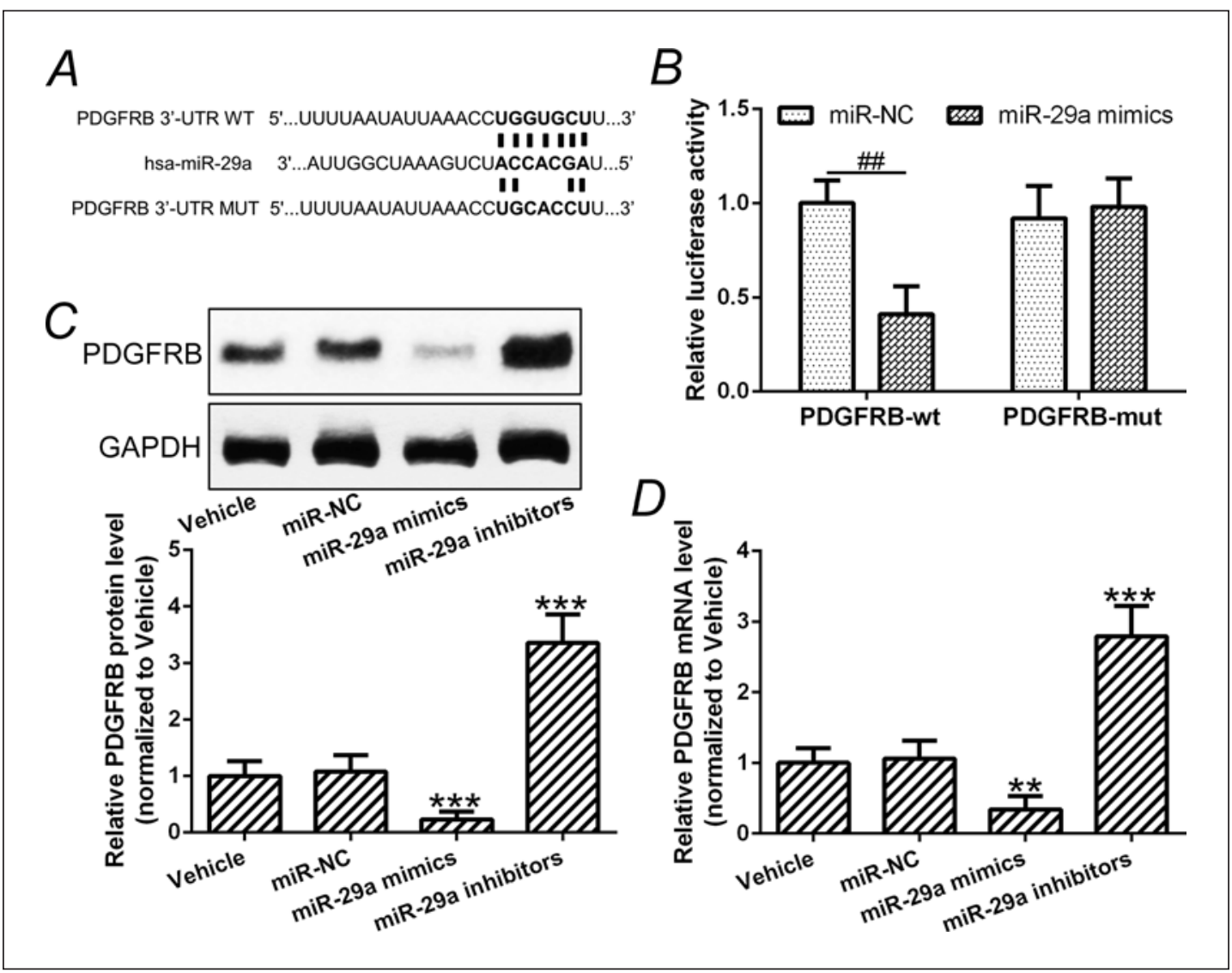

Fig. 3. PDGFRB was identified as a target of miR-29a in HASMCs. A The suspected binding of miR-29a with the wild-type 3'-UTR region of PDGFRB mRNA is shown. A mutated 3'-UTR of PDGFRB is also shown. B Luciferase reporter assay to elucidate the targeting relationship between miR-29a and PDGFRB in HEK 293T cells. C Western blotting was used to measure the PDGFRB protein expression in HASMCs transfected with miR-29a mimics or miR-29a inhibitors for $24 \mathrm{~h}$, and relative expression was normalized to vehicle group. D qRT-PCR was used to measure the PDGFRB mRNA expression in HASMCs transfected with miR-29a mimics or miR-29a inhibitors for $24 \mathrm{~h}$, and relative expression was normalized to vehicle group. Data are presented as mean \pm SD. ${ }^{* *} p<0.01$ and ${ }^{* * *} p<0.001$ compared to control. ${ }^{\# \#} p<0.01$ between the comparison. PDGFRB, platelet-derived growth factor receptor $B$.

PDGF-BB than the miR-NC and blank controls ( $p<0.001$, Fig. $2 \mathrm{C}$ ). These results indicated the successful establishment of ASO cell model, which could serve as useful tool to investigate the role of miR-29a.

miR-29a Targets the 3'-UTR of PDGFRB and Downregulates Its Expression

Bioinformatics prediction via TargetScan website identified PDGFRB as a potential target of miR-29a. The putative target sites for hsa-miR-29a at the 3'-UTR of PDGFRB were conserved (Fig. 3A). As shown in Figure 3B, in comparison with the miR-NC, the miR-29a mimics significantly reduced the relative luciferase activity in the wild-type PDGFRB group $(p<0.01)$, but did not present significant difference in the mutant-type PDGFRB (Fig. 3B), suggesting the direct binding between miR-29a and the 3 '-UTR of PDGFRB.

To further investigate the functional binding between PDGFRB and miR-29a in HASMCs, HASMCs were transfected with miR-NR, miR-29a mimics, and miR-29a inhibitor, and the PDGFRB expression levels were determined. As shown in Figure 3C, the protein level of 
Kidney

Blood Pressure

Research

\begin{tabular}{|c|c|}
\hline Kidney Blood Press Res 201 & :1219-1232 \\
\hline DOI: 10.1159/000502649 & $\begin{array}{l}\text { (c) } 2019 \text { The Author(s). Published by S. Karger AG, Basel } \\
\text { www.karger.com/kbr }\end{array}$ \\
\hline
\end{tabular}

Wang et al.: The Role of miR-29a in ASO
Fig. 4. miR-29a-regulated PDGFRB expression in PDGF-BB-stimulated HASMCs. A qRT-PCR was used to measure the PDGFRB mRNA expression in HASMCs transfected with miR-29a mimics or miR-29a inhibitors for $24 \mathrm{~h}$, followed by PDGF-BB stimulation for $24 \mathrm{~h}$, and relative expression was normalized to vehicle group. B Western blotting was used to measure the PDGFRB protein expression in HASMCs transfected with miR-29a mimics or miR-29a inhibitors for $24 \mathrm{~h}$, followed by PDGF-BB stimulation for $24 \mathrm{~h}$, and relative expression was normalized to vehicle group. Data are presented as mean \pm SD. $* p<0.05,{ }^{* *} p<0.01$ and ${ }^{* * *} p$ $<0.001$ compared to control. ${ }^{\#} p<$ 0.05 , ${ }^{\# \#} p<0.01$ between the comparison. PDGFRB, platelet-derived growth factor receptor B; PDGF$\mathrm{BB}$, platelet-derived growth factor-BB.

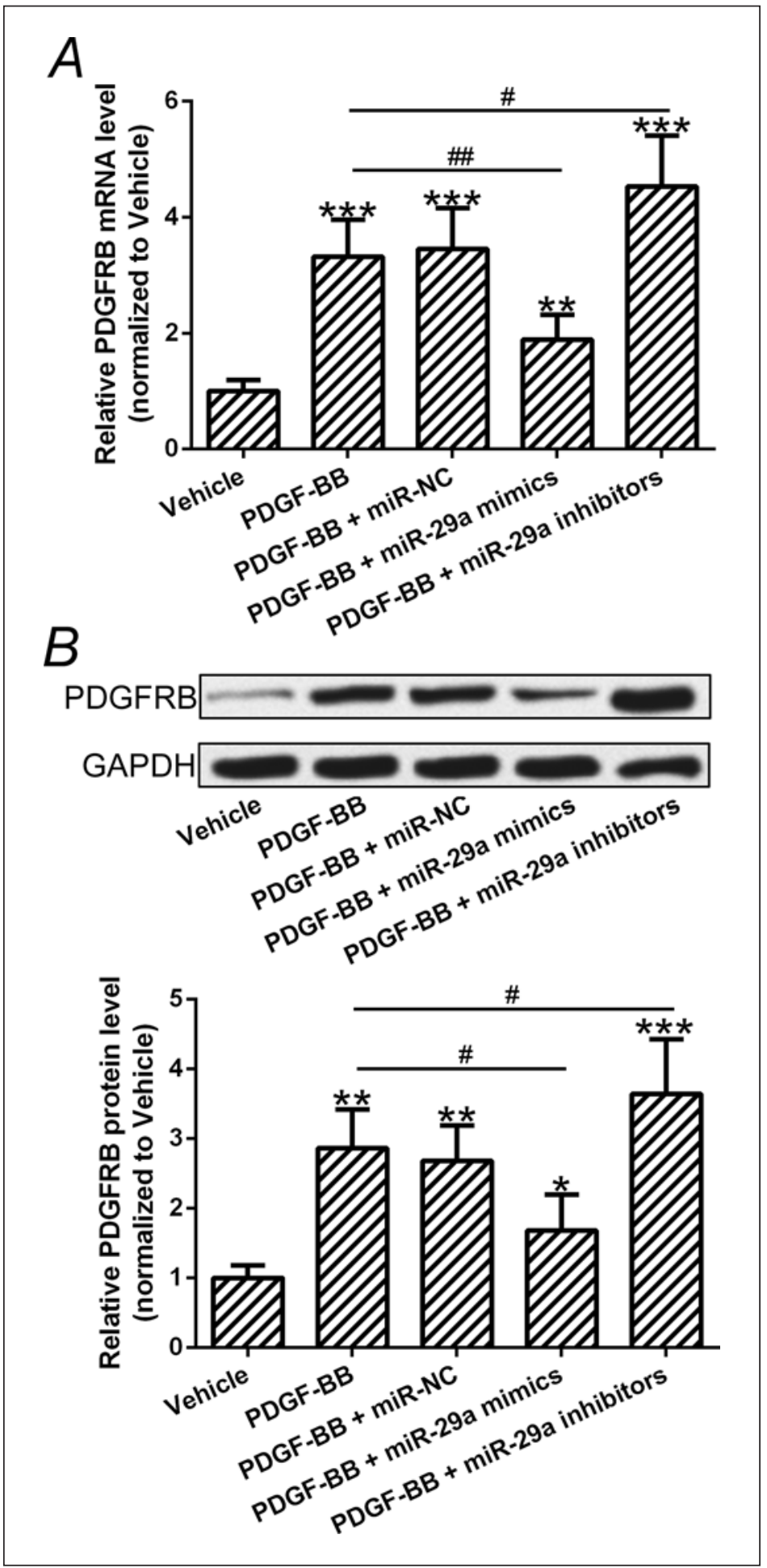

PDGFRB was significantly decreased following miR-29a mimics transfection $(p<0.001)$, and PDGFRB was significantly upregulated in the HASMCs transfected with a miR-29a inhibitors $(p<0.001)$. The relative mRNA level of PDGFRB showed similar changes as protein level by miR-29a mimics and inhibitor transfections (Fig. 3D). These results demonstrated that miR-29a negatively regulates the expression of PDGFRB in HASMCs. 


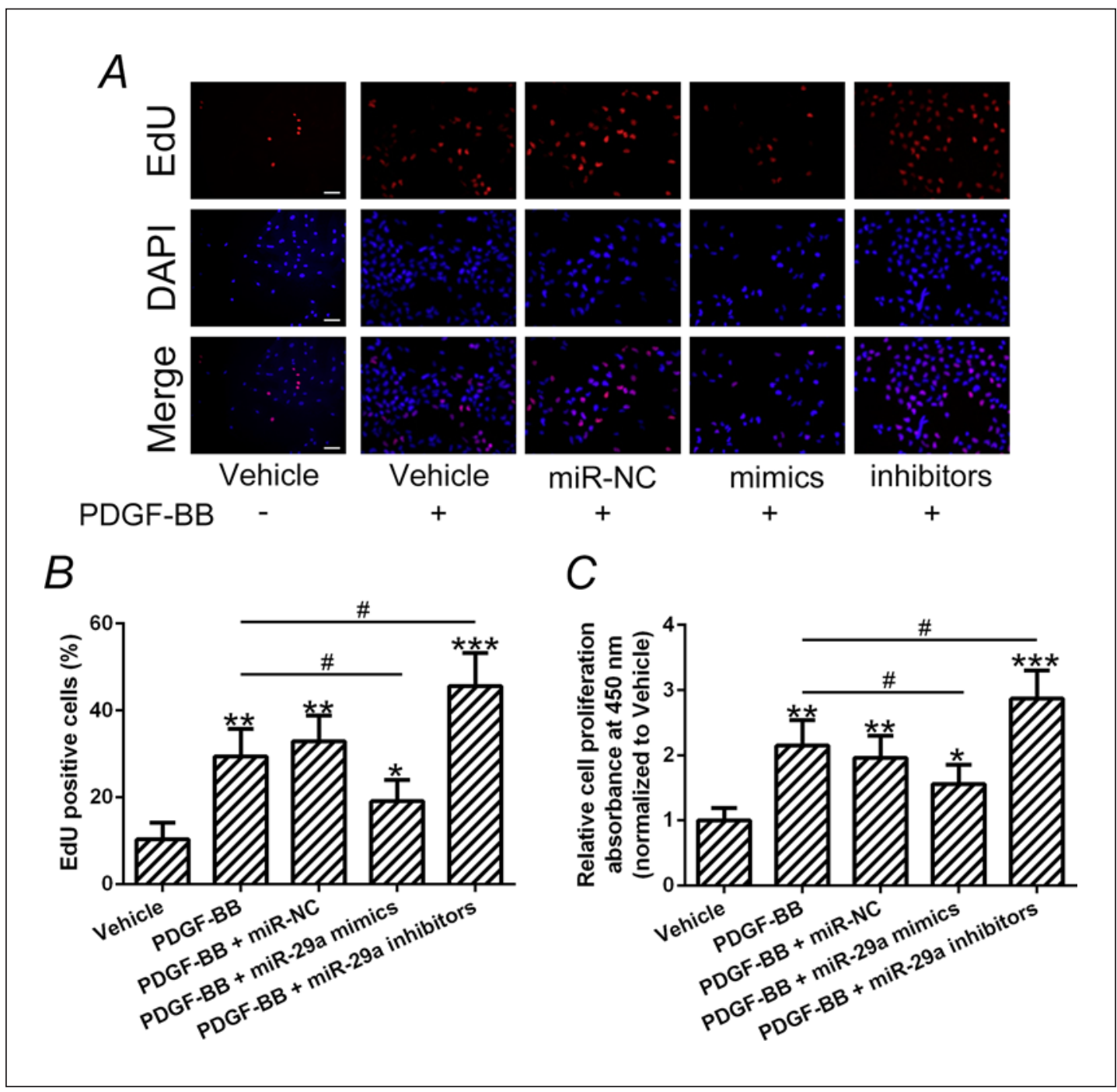

Fig. 5. miR-29a-regulated proliferation of PDGF-BB-stimulated HASMCs. HASMCs were transfected with miR-29a mimics or miR-29a inhibitors for $24 \mathrm{~h}$, followed by PDGF-BB stimulation for $24 \mathrm{~h}$. HASMC proliferation was determined by EdU assay (A, B) and cell counting kit-8 assay (C). Scale bar: $50 \mu \mathrm{m}$. Data are presented as mean \pm SD. ${ }^{*} p<0.05,{ }^{* *} p<0.01$, and ${ }^{* * *} p<0.001$ compared to control. ${ }^{*} p<0.05$ between the comparison. PDGF-BB, platelet-derived growth factor-BB.

miR-29a Regulates PDGFRB Expression in PDGF-BB-Stimulated HASMCs

To further investigate the interaction between miR-29a and PDGFRB expression, miR-NC, miR-29a mimics, and miR-29a inhibitors were transfected into the PDGF-BB-stimulated HASMCs. As seen in Figure 4A, PDGF-BB stimulation significantly increased the expression of PDGFRB in HASMCs compared to the vehicle control $(p<0.001)$, and overexpression of miR-29a by miR-29a mimics could significantly inverse this change caused by PDGF-BB treatment $(p<0.01)$. Likewise, the miR-29a inhibitors significantly increased the expression of PDGFRB in the PDGF-BB-stimulated HASMCs $(p<0.001)$. This changes of PDGFRB expression were verified at protein level by western blot assay (Fig. 4B). These observations showed that miR-29a negatively regulates the expression of PDGFRB at both mRNA and protein levels in PDGF-BB-stimulated HASMCs. 


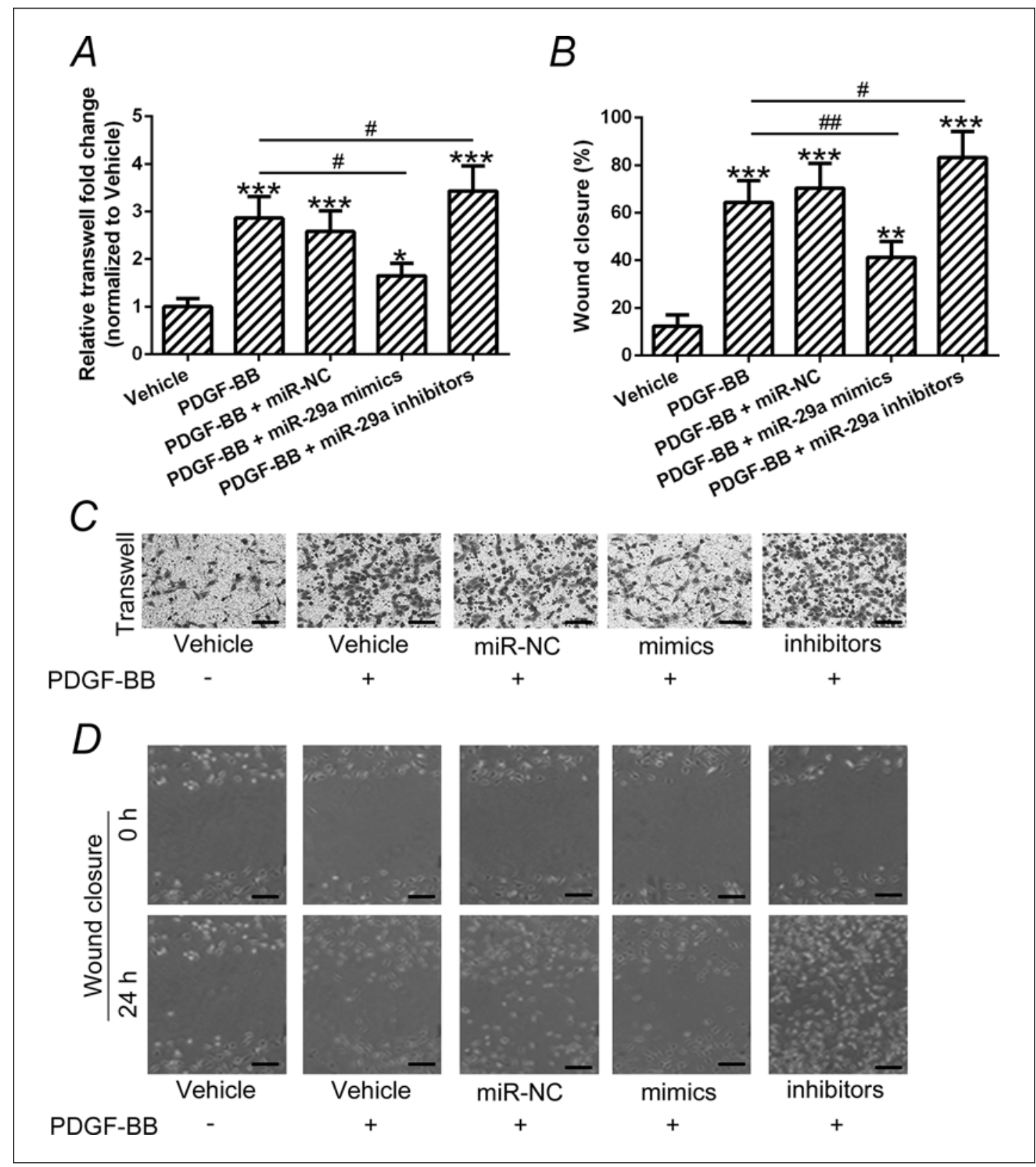

Fig. 6. miR-29a-regulated migration of PDGF-BB-stimulated HASMCs. HASMCs were transfected with miR-29a mimics or miR-29a inhibitors for $24 \mathrm{~h}$, followed by PDGF-BB stimulation for $24 \mathrm{~h}$. HASMC migration was determined by Transwell assay $(\mathbf{A}, \mathbf{C})$ and wound closure assay (B, D). Scale bar: $200 \mu \mathrm{m}$. Data are presented as mean \pm SD. ${ }^{*} p<0.05,{ }^{* *} p<0.01$, and ${ }^{* * *} p<0.001$ compared to control. ${ }^{\#} p<0.05$, ${ }^{\# \#} p<0.01$ between the comparison. PDGF-BB, platelet-derived growth factor-BB.

miR-29a Inhibits Proliferation in PDGF-BB-Stimulated HASMCs

To determine whether miR-29a affect the HASMC proliferation induced by PDGF-BB, miR-NC, miR-29a mimics, and miR-29a inhibitors were transfected into HASMCs, and proliferation was assessed via EdU and CCK-8 assays. As shown in Figure 5, these assays demonstrated that PDGF-BB stimulation significantly increased the proliferation of HASMCs indicated by the higher percentage of EdU-positive cells (Fig. 5A, B) and higher absorbance at 450 $\mathrm{nm}$ (Fig. 5C). Significantly, the upregulation of miR-29a by miR-29a mimics significantly 


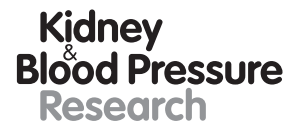

Research \begin{tabular}{l|l}
\hline Kidney Blood Press Res 2019;44:1219-1232 \\
\hline DOI: 10.1159/000502649 & $\begin{array}{l}\text { @ 2019 The Author(s). Published by S. Karger AG, Basel } \\
\text { www.karger.com/kbr }\end{array}$ \\
\hline
\end{tabular}

Wang et al.: The Role of miR-29a in ASO

inhibited HASMC proliferation compared with the control group $(p<0.001)$, and miR-29a inhibitors significantly promoted the proliferation of HASMCs. These results suggested that miR-29a inhibited HASMCs proliferation in vitro.

\section{miR-29a Inhibits Migration in PDGF-BB-Treated HASMCs}

To detect the effects of miR-29a on the HASMC migration induced by PDGF-BB, miR-NC, miR-29a mimics, and miR-29a inhibitors were transfected into induced HASMCs. Cell migration induced by PDGF-BB was compared in Transwell devices. As shown in Figure 6, the miR-29a mimics significantly inhibited PDGF BB-induced HASMCs migration in comparison with the control group ( $p<0.001$, Fig. $6 \mathrm{~A}, \mathrm{C})$, and the miR-29a inhibitors significantly enhanced PDGF BB-induced HASMCs migration when compared to the control group. Similar results were observed in the wound closure assay ( $p<0.001$; Fig. 6B, D). In addition, we compared the effects of miR-29a mimics and si-PDGFRB (Sangon Biotech, Shanghai, China), and the results showed that miR-29a mimics showed similar extent of inhibition on the proliferation and migration as the blockage of PDGFBR (online suppl. Fig. S1). These results demonstrated that miR-29a negatively regulates the in vitro migration of HASMCs.

\section{Discussion}

ASO is one of the most miserable vascular disorders with high morbidity and mortality, seriously impairing the life quality of the patients [14]. There are a great number of studies that have investigated the underlying pathological mechanisms of this disorder. Among them, it has been well established that the HASMCs play pivotal role in arteriosclerosis and vascular stenosis [15]. HASMCs are the main components of the vascular wall. In spite of the development of the understanding in vascular smooth muscle biology [16], the molecular mechanisms about how HASMCs contribute to the pathogenesis of ASO remain elusive. It has been revealed that miRNAs mediate a great variety of physiological and pathological processes by regulating the target genes at a post-transcriptional level [17], and tons of studies have shown that miRNAs serve pivotal roles in cardiovascular systems [18]. In this study, we demonstrated that miR-29a expression was remarkably downregulated in the arterial walls of ASO patients compared with the arterial walls from normal donors. We observed for the first time that miR-29a inhibited the proliferation and migration in PDGF-BB-induced HASMCs, which was highly related to the expression of PDGFRB.

To be noted, the tissue specificity is an important feature of miRNA expression [19]. Consistently, in this study, our qRT-PCR and western blot results supported that miR-29a expression levels were significantly downregulated in ASO arterial walls when compared with the normal arterial walls. In a more detailed way, the ASO and normal arterial walls were dissected into 3 constituent layers including adventitia, media, and endothelium. We found that the expression of miR-29a was only significantly changed in the media, but not in adventitia and endothelium. It has been known that media contain arterial smooth muscle cell. In order to verify the essential participation of arterial smooth muscle cells, primary HASMCs were isolated and cultured to investigate the expression of miR-29a. Our results showed that miR-29a expression was decreased in HASMCs stimulated with PDGF-BB, compared with vehicle treated cells. All these findings indicated that HASMCs may function as the main effector cells of miR-29a in the pathogenesis of ASO.

Particularly, miR-29a expression is highly relevant to the proliferating cells that were stimulated by PDGF. We used PDGF-BB to treat primary HASMCs and observed that PDGF-BB could lower the expression of miR-29a and increase the expression of PDGFRB in HASMCs. It has been previously showed that PDGF regulates the activation of signal transducer and acti- 


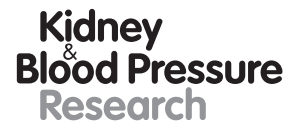

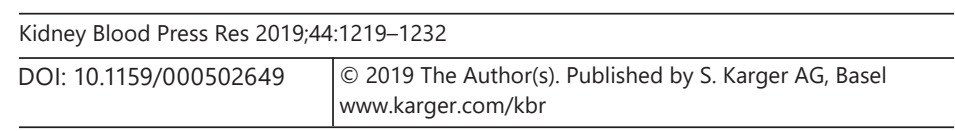

Wang et al.: The Role of miR-29a in ASO

vator of transcription 1 [18], which has been implicated as a transcriptional regulator of the miR-29ab1 cluster [20]. PDGFs were discovered in 1974 and have been extensively reported to have effects on the proliferation and migration of VSMCs [21]. However, it is still not clear how the expression of PDGFs and their receptors in eccentric atherosclerotic lesions are regulated. Interestingly enough, our study showed that PDGF-BB downregulated the expression of miR-29a but enhanced the expression of PDGFRB. It has been reported that aggregating platelets or leukocytes result in high PDGF expression in atherosclerosis [21, 22]. According to our in vitro model, we speculated that this high PDGF expression decreased miR-29a expression and then neutralized the inhibition on PDGFRB expression.

PDGFRB is a receptor of PDGF that binds to PDGF-B and PDGF-D [23], which is a kind of receptor tyrosine kinases, possesses intrinsic protein tyrosine kinase activity in the cytoplasmic domains [24]. PDGFRB has an important role in atherosclerosis, and it has been reported that its activity is related to the promotion on the migration and proliferation of VSMCs $[25,26]$. In this current study, we predicted and verified that PDGFRB is a direct target gene of miR-29a. Specifically, we revealed that the expression of PDGFRB at both mRNA and protein levels was upregulated in the arterial wall of ASO patients, which was negatively related to the downregulation of miR-29a in the same clinical samples. Further, we confirmed that miR-29a was able to bind with the 3'-UTR of PDGFRB by luciferase reporter assay, indicating that PDGFRB is a direct target of miR-29a. In addition, we found that the overexpression of miR-29a by transfection of miR-29a mimics could significantly decrease the relative PDGFRB expression, and transfection of miR-29a inhibitors increases the expression of PDGFRB in HASMCs, suggesting the negative regulation of miR-29a on PDGFRB expression.

Given the important role of PDGFRB in HASMCs, we further explored the modulation of miR-29a on the proliferation and migration of HASMCs. The functions of miR-29a in the physiological and pathological processes in ASO were worthy for deeper study. To explore the details of the regulation of miR-29a on HASMCs, HASMCs cell proliferation was investigated by CCK-8 and EdU assays, and cell migrations were evaluated by Transwell and wound closure assays. Our results showed that miR-29a was able to inhibit the proliferation and migration in PDGF-BB-induced HASMCs. Specifically, miR-29a mimics significantly decreased the proliferation of HASMCs enhanced by PDGF-BB compared with the miR-NC control, whereas the miR-29a inhibitors promoted the cell proliferation of HASMCs. miR-29a plays an important role in the regulation of tumor cell migration and invasion [27]. For instance, a negative regulatory relationship was observed between miR-29a and p42.3 expression in cell lines and clinical tissue samples of gastric cancer [24]. Another study demonstrated that the expression of miR-29a could decrease cell proliferation in gastric cancer via diluting the level of Cyclindependent kinase (CDK) 2, CDK4, and CDK6 [28]. In combining with these background literatures, we concluded that the inhibition of miR-29a on the proliferation and migration of HASMCs was through the regulation of PDGFRB expression.

In conclusion, our study showed that miR-29a is downregulated in the arterial wall of ASO and in the PDGF-BB-stimulated HASMCs. miR-29a binds target gene PDGFRB and inhibits the proliferation and migration of PDGF-BB-induced HASMCs. Our findings provided new insights that the miR-29a/PDGFRB axis could be developed as a potential target for ASO therapeutically.

This study showed that miR-29a is downregulated in the arterial wall of ASO patients, as well as in the PDGF-BB-stimulated HASMCs. This alteration of miR-29a could upregulate target genes PDGFRB and inhibits the proliferation and migration of HASMCs. These findings discovered new mechanisms of ASO pathogenesis, and the miR-29a/PDGFRB axis could serve as potential target for the therapy of ASO. 


\section{Statement of Ethics}

The authors have no ethical conflicts to disclose.

\section{Disclosure Statement}

The authors declare that there is no conflict of interest.

\section{Funding Source}

The study was supported by Shandong Excellent Young and Middle-aged Scientist Research Award Fund (SQ20160033).

\section{References}

1 Liu J, Li W, Wang S, Wu Y, Li Z, Wang W, et al. Mir-142-3p attenuates the migration of cd4(+) t cells through regulating actin cytoskeleton via rac1 and rock2 in arteriosclerosis obliterans. PLoS One. 2014;9(4):e95514.

2 Horie T, Kimura T, Ono K. Emerging novel biomarkers for arteriosclerosis obliterans. J Atheroscler Thromb. 2016;23(2):171-2.

3 Setacci C, Castelli P, Chiesa R, Grego F, Simoni GA, Stella A, et al. Restenosis: A challenge for vascular surgeon. J Cardiovasc Surg (Torino). 2012;53:735-46.

4 Bartel DP. Micrornas: Genomics, biogenesis, mechanism, and function. Cell. 2004;116(2):281-97.

5 Wang J, Chen J, Sen S. Microrna as biomarkers and diagnostics. J Cell Physiol. 2016;231(1):25-30.

6 He XM, Zheng YQ, Liu SZ, Liu Y, He YZ, Zhou XY. Altered plasma micrornas as novel biomarkers for arteriosclerosis obliterans. J Atheroscler Thromb. 2016;23(2):196-206.

7 Huang S, Chen Z, Wu W, Wang M, Wang R, Cui J, et al. Microrna-31 promotes arterial smooth muscle cell proliferation and migration by targeting mitofusin-2 in arteriosclerosis obliterans of the lower extremitie. Exp Ther Med. 2018;15:633-40.

8 Mourelatos Z, Dostie J, Paushkin S, Sharma A, Charroux B, Abel L, et al. Mirnps: A novel class of ribonucleoproteins containing numerous micrornas. Genes Dev. 2002;16(6):720-8.

9 Mazzoccoli L, Robaina MC, Apa AG, Bonamino M, Pinto LW, Queiroga E, et al. Mir-29 silencing modulates the expression of target genes related to proliferation, apoptosis and methylation in burkitt lymphoma cells. J Cancer Res Clin Oncol. 2018;144(3):483-97.

10 Xiao Z, Wang Y, Ding H. Xpd suppresses cell proliferation and migration via mir-29a-3p-mdm2/pdgf-b axis in hcc. Cell Biosci. 2019 Jan; 9:6.

11 Chen KC, Wang YS, Hu CY, Chang WC, Liao YC, Dai CY, et al. Oxldl up-regulates microrna-29b, leading to epigenetic modifications of mmp-2/mmp-9 genes: A novel mechanism for cardiovascular diseases. FASEB J. 2011;25(5):1718-28.

12 Roncarati R, Viviani Anselmi C, Losi MA, Papa L, Cavarretta E, Da Costa Martins P, et al. Circulating mir-29a, among other up-regulated micrornas, is the only biomarker for both hypertrophy and fibrosis in patients with hypertrophic cardiomyopathy. J Am Coll Cardiol. 2014;63(9):920-7.

13 Wang M, Li W, Chang GQ, Ye CS, Ou JS, Li XX, et al. Microrna-21 regulates vascular smooth muscle cell function via targeting tropomyosin 1 in arteriosclerosis obliterans of lower extremities. Arterioscler Thromb Vasc Biol. 2011;31(9):2044-53.

14 Diehm C, Allenberg JR, Pittrow D, Mahn M, Tepohl G, Haberl RL, et al. Mortality and vascular morbidity in older adults with asymptomatic versus symptomatic peripheral artery disease. Circulation. 2009;120(21):205361.

15 Kim J, Zhang L, Peppel K, Wu JH, Zidar DA, Brian L, et al. Beta-arrestins regulate atherosclerosis and neointimal hyperplasia by controlling smooth muscle cell proliferation and migration. Circ Res. 2008;103(1):70-9.

16 Hao H, Gabbiani G, Bochaton-Piallat ML. Arterial smooth muscle cell heterogeneity: implications for atherosclerosis and restenosis development. Arterioscler Thromb Vasc Biol. 2003;23(9):1510-20.

17 Ambros V. The functions of animal micrornas. Nature. 2004;431(7006):350-5.

18 Vignais ML, Sadowski HB, Watling D, Rogers NC, Gilman M. Platelet-derived growth factor induces phosphorylation of multiple jak family kinases and stat proteins. Mol Cell Biol. 1996;16(4):1759-69.

19 Bushati N, Cohen SM. Microrna functions. Annu Rev Cell Dev Biol. 2007;23(1):175-205. 
Smith KM, Guerau-de-Arellano M, Costinean S, Williams JL, Bottoni A, Mavrikis Cox G, et al. Mir-29ab1 deficiency identifies a negative feedback loop controlling th1 bias that is dysregulated in multiple sclerosis. J Immunol. 2012 Aug;189(4):1567-76.

21 Ross R, Glomset J, Kariya B, Harker L. A platelet-dependent serum factor that stimulates the proliferation of arterial smooth muscle cells in vitro. Proc Natl Acad Sci USA. 1974;71(4):1207-10.

22 Heldin CH, Westermark B. Mechanism of action and in vivo role of platelet-derived growth factor. Physiol Rev. 1999;79(4):1283-316.

23 Hart CE, Forstrom JW, Kelly JD, Seifert RA, Smith RA, Ross R, et al. Two classes of pdgf receptor recognize different isoforms of pdgf. Science. 1988;240(4858):1529-31.

24 Cui Y, Su WY, Xing J, Wang YC, Wang P, Chen XY, et al. Mir-29a inhibits cell proliferation and induces cell cycle arrest through the downregulation of p42.3 in human gastric cancer. PLoS One. 2011;6(10):e25872.

25 Boucher P, Liu P, Gotthardt M, Hiesberger T, Anderson RG, Herz J. Platelet-derived growth factor mediates tyrosine phosphorylation of the cytoplasmic domain of the low density lipoprotein receptor-related protein in caveolae. J Biol Chem. 2002;277(18):15507-13.

26 Boucher P, Gotthardt M, Li WP, Anderson RG, Herz J. Lrp: role in vascular wall integrity and protection from atherosclerosis. Science. 2003;300(5617):329-32.

27 Wang JY, Zhang Q, Wang DD, Yan W, Sha HH, Zhao JH, et al. Mir-29a: A potential therapeutic target and promising biomarker in tumors. Biosci Rep. 2018;38(1):38.

28 Zhao Z, Wang L, Song W, Cui H, Chen G, Qiao F, et al. Reduced mir-29a-3p expression is linked to the cell proliferation and cell migration in gastric cancer. World J Surg Oncol. 2015 Mar;13:101. 